\title{
THE RINGS OF URANUS: RESULTS OF THE 10 APRIL 1978 OCCULTATION
}

\author{
P. D. NICHOLSON \\ Division of Geological and Planetary Sciences, California Institute of Technology, Pasadena, California 91125
}

S. E. PERSSON

Hale Observatories, California Institute of Technology, Carnegie Institution of Washington, Pasadena, California 91101

\section{K. MATTHEWS}

California Institute of Technology, Pasadena, California 91125

\section{P. GOLDREICH}

Division of Geological and Planetary Sciences, California Institute of Technology, Pasadena, California 91125

\section{G. NeUGebaueR}

Hale Observatories, California Institute of Technology, Carnegie Institution of Washington, Pasadena, California 91101 Received 26 June 1978

\begin{abstract}
Observations of the 10 April 1978 stellar occultation by the rings of Uranus are presented. Nine rings were observed and their radii and widths are calculated. Rings $\eta, \gamma$, and $\delta$ are found to be most likely circular and coplanar, in agreement with previous analyses; the remaining rings are either noncircular or slightly inclined. The width of the $\epsilon$ ring is a linear function of its radius from the center of Uranus, projected onto the satellites' orbital plane; this suggests that it forms one continuous noncircular ring. The optical depth profile of the $\epsilon$ ring has not changed significantly since March 1977. A model of this ring which fits all available observations adequately is that of a uniformly precessing Keplerian ellipse coplanar with the satellites' orbits. This model permits predictions of the radius and width of the $\epsilon$ ring for future occultations. The precession rate is used to determine $J_{2}$ for Uranus, on the assumption that precession is caused solely by the planetary oblateness and not by satellite-ring interactions.
\end{abstract}

\section{INTRODUCTION}

The Uranus ring system was discovered on 10 March 1977, by Elliot, Dunham, and Mink (1977), during observations of an occultation by the planet of the star SAO $158687(V \sim 8.8)$. Other observers obtained less complete data, but nevertheless confirmed the existence of four narrow $(\$ 10 \mathrm{~km})$, essentially circular, coplanar rings, christened $\alpha, \beta, \gamma$, and $\delta$, and the wider $(\sim 100 \mathrm{~km})$, noncircular or inclined $\epsilon$ ring (Millis, Wasserman, and Birch 1977; Bhattacharyya and Kuppuswamy 1977a; Churms 1977; Zellner 1977; Mahra and Gupta 1977). Subsequently, Elliot et al. (1978) have identified four more rings: $\eta, 4,5$, and 6. On 23 December 1977, Millis and Wasserman (1978) observed a partial occultation by the ring system of $\mathrm{BD}-15^{\circ} 3969(V=10.4)$. Despite poor observing conditions near dawn, they detected and timed occultations by the $\epsilon, \delta, \gamma$, and (possibly) $\alpha$ rings on one side of the planet.

Predictions of further occultations by the Uranian system were made by Klemola and Marsden (1977). This paper reports observations of the events of 4 and 10
April 1978, designated Nos. 4 and 5, respectively, by Klemola and Marsden. Both observations were made at a wavelength of $2.2 \mu \mathrm{m}$, where a strong methane band greatly depresses the reflected light from the planet. Table I gives the $V$ and $2.2-\mu \mathrm{m}$ magnitudes of Uranus and the two stars.

Note: On 8 January 1978, the $2.2-\mu \mathrm{m}$ magnitude of Uranus was measured to be $12.9 \pm 0.2$ with a 5 " diameter aperture which excluded any direct contribution by the rings (diameter $\sim 8^{\prime \prime}$ ). Subsequent observations on 7 May 1978 with a 16" aperture gave $[2.2-\mu \mathrm{m}]=11.6 \pm 0.2$. Joyce et al. (1977), using an unspecified aperture size, have also reported a $2.2-\mu \mathrm{m}$ magnitude of $11.5-12.0$ for Uranus. These results suggest that the integrated $2.2-\mu \mathrm{m}$ magnitude of the ring system is $\sim 12.0$. If the average effective width of the rings is $90 \mathrm{~km}$, this implies a geometric albedo at this wavelength of 0.025 .

The results from the 10 April 1978 occultation are the main subject of this paper. The 4 April 1978 occultation yielded little meaningful data on the rings. 
TABLE I. Magnitude of Uranus and occulted stars.

\begin{tabular}{ccc}
\hline \hline & \multicolumn{2}{c}{ Magnitude } \\
\cline { 2 - 3 } Object & $V$ & {$[2.2 \mu \mathrm{m}]$} \\
\hline Uranus & 5.5 & $12.9 \pm 0.2^{\mathrm{a}}$ \\
Star No. 4 & $13.4^{\mathrm{b}}$ & 11.9 \\
Star No. 5 & $11.6^{\mathrm{b}}$ & 10.1 \\
\hline \hline
\end{tabular}

a See note to Sec. I.

b Liller (1977).

\section{OBSERVATIONS}

The observations were made using an infrared photometer mounted on the 2.5-m DuPont telescope at Las Campanas Observatory in Chile. A standard $K$ filter $\left(\lambda_{0}\right.$ $=2.20 \mu \mathrm{m} ; \Delta \lambda=0.4 \mu \mathrm{m})$ was used with an InSb detector cooled to $55 \mathrm{~K}$. Sky subtraction was performed by chopping at $15 \mathrm{~Hz}$ to a secondary beam located $30^{\prime \prime}$ to the north.

Star No. 5 is considerably brighter than the Uranus system (planet + rings) at $2.2 \mu \mathrm{m}$-bright enough to give a signal-to-noise ratio of $\sim 10$ with a time resolution of $\sim 0.1 \mathrm{~s}$. The noise was due almost completely to background radiation from the telescope and sky, and it was thus necessary to use a small focal plane aperture centered on the star. An aperture diameter of 7..5 was selected to reduce thermal background noise without introducing noise from guiding and seeing effects. Fortunately, the observing conditions were excellent and the seeing less than 1". The star was centered by finding the half-power points of the $2.2-\mu \mathrm{m}$ signal $1 \mathrm{~h}$ before the first $\epsilon$ ring occultation, and centering was maintained thereafter by using an offset guider/Quantex television system. The chopped signal from the detector was demodulated in a conventional lock-in amplifier and recorded on a strip chart, with an overall system time constant of $0.1 \mathrm{~s}$. Absolute timing accurate to $<0.3 \mathrm{~s}$ was obtained from WWV.

Star No. 4 is of comparable brightness to the Uranus system at $2.2 \mu \mathrm{m}$, and the signal-to-noise ratio at a time resolution of $0.1 \mathrm{~s}$ was $\sim 1$. The dawn sky rendered guiding impossible about 10 min after planetary emersion.

\section{RESULTS}

The apparent tracks of the two occulted stars behind Uranus and its rings are shown in Fig. 1. For comparison, the 10 March 1977 and 23 December 1977 tracks are shown also. On 10 April 1978 the inclination of the ring plane (assumed to be the satellites' orbit plane) to the sky was $32^{\circ} 85$.

On 10 April 1978, two complementary sets of nine ring occultations were recorded. All of the ring occultations, as recorded on the strip chart, are displayed in Fig. 2. Analysis of the data (Sec. IV $a$ ) reveals that these nine rings correspond in radius to the nine rings described by Elliot et al. (1978), and they have been identified accordingly. In an initial report of these observations
(Persson et al. 1978), the designations $\theta$, $\iota$, and $\kappa$ were used for the rings referred to as 4, 5, and 6, respectively, by Elliot et al. (1978). To avoid confusion, the terminology of the latter authors is used throughout this paper. Table II gives the times, durations, and fractional depths of the occultations. The depths have not been corrected for the presence of any planetary or ring component in the observed $2.2-\mu \mathrm{m}$ flux, but this component is $\leq 10 \%$ of the total. Relative timing is accurate to $\sim 0.1 \mathrm{~s}$, and absolute timing to $<0.3 \mathrm{~s}$. Continuöus observations were obtained between $5^{\mathrm{h}} 09^{\mathrm{m}}$ and $7^{\mathrm{h}} 05^{\mathrm{m}}$ UT, except for $\leq 30$-s gaps at $6^{\mathrm{h}} 39^{\mathrm{m}}$ and $6^{\mathrm{h}} 48^{\mathrm{m}}$, but the data do not reveal any further significant occultations which might correspond to those reported by Churms (1977) and Millis and Wasserman (1978) during the March and December 1977 events. The record shows no evidence at the 5\% level of any smoothly varying background absorption. No planetary occultation occurred, consistent with the prediction.

On 4 April 1978, a planetary occultation with a duration between half-light points of $44 \mathrm{~min} 48 \pm 10 \mathrm{~s}$ occurred at Las Campanas. The midpoint of this event, at $10^{\mathrm{h}} 09^{\mathrm{m}} 50 \pm 10^{\mathrm{s}} \mathrm{UT}$, was 4 min ahead of the prediction by Klemola and Marsden (1977). Only one convincing ring occultation, at $9^{\mathrm{h}} 31^{\mathrm{m}} 00^{\mathrm{s}} \mathrm{UT}$, is identifiable in the very noisy record. This occultation lasted $\sim 1 \mathrm{~s}$ and presumably represents the $\epsilon$ ring. Guiding became impossible in the dawn twilight before the predicted time of the second $\epsilon$-ring occultation. The poor signal-to-noise ratio of the record precludes any useful analysis of the plan-

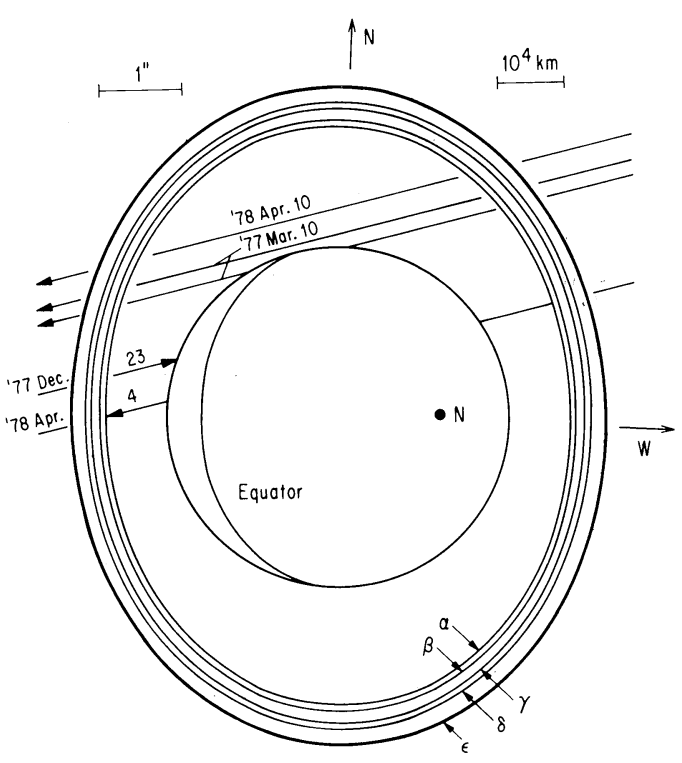

FIG. 1. 1977-1978 aspect of Uranus and rings. The $\eta$ ring lies between $\beta$ and $\gamma$, while 4,5 , and 6 are just inside $\alpha$. The straight lines show the apparent paths of the occulted stars behind the ring system, as they were observed from the various earth stations. The upper track labeled 10 March 1977 corresponds to Perth, Western Australia, and the lower track to the Kuiper Airborne Observatory. 

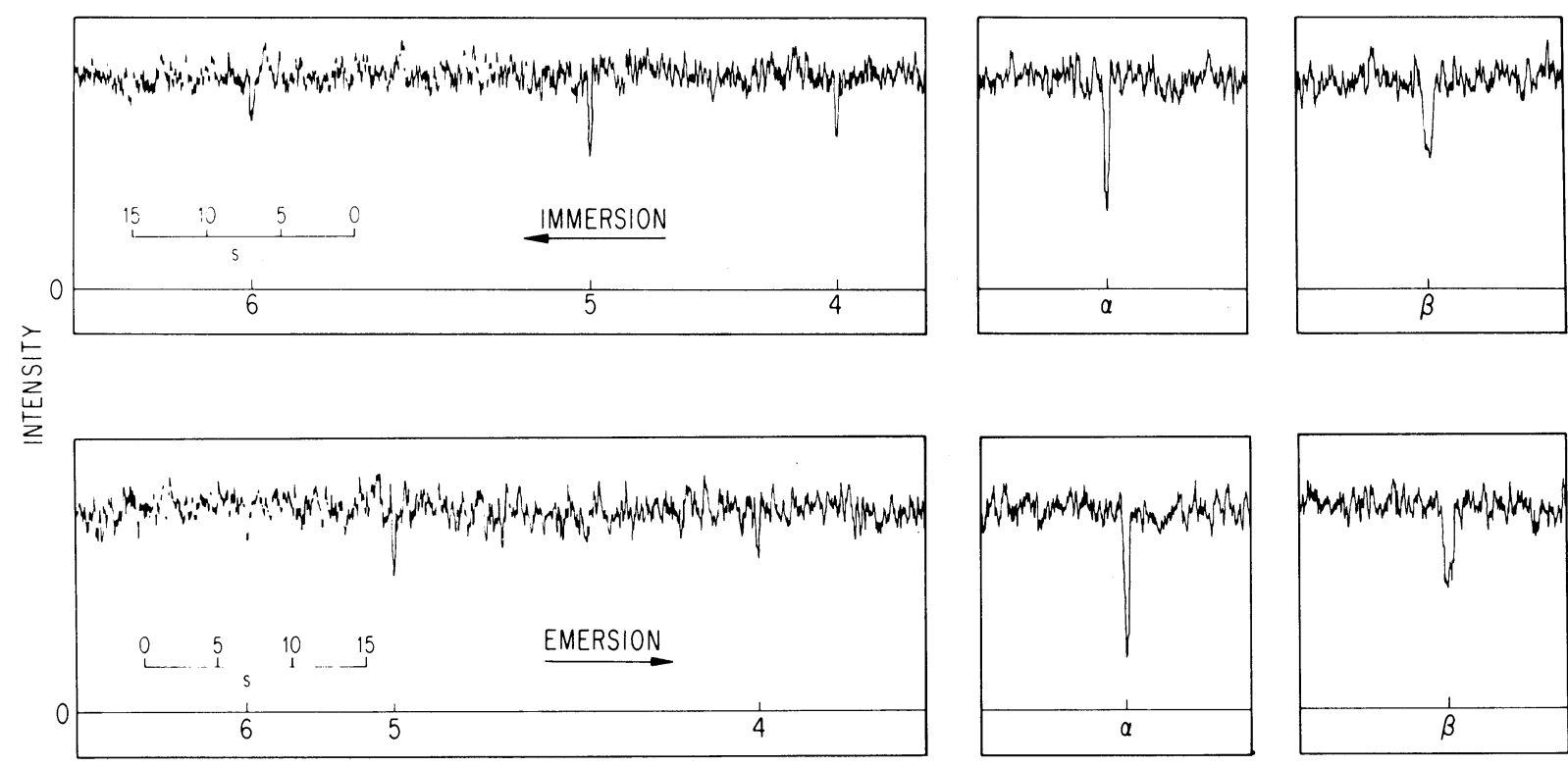

(a)
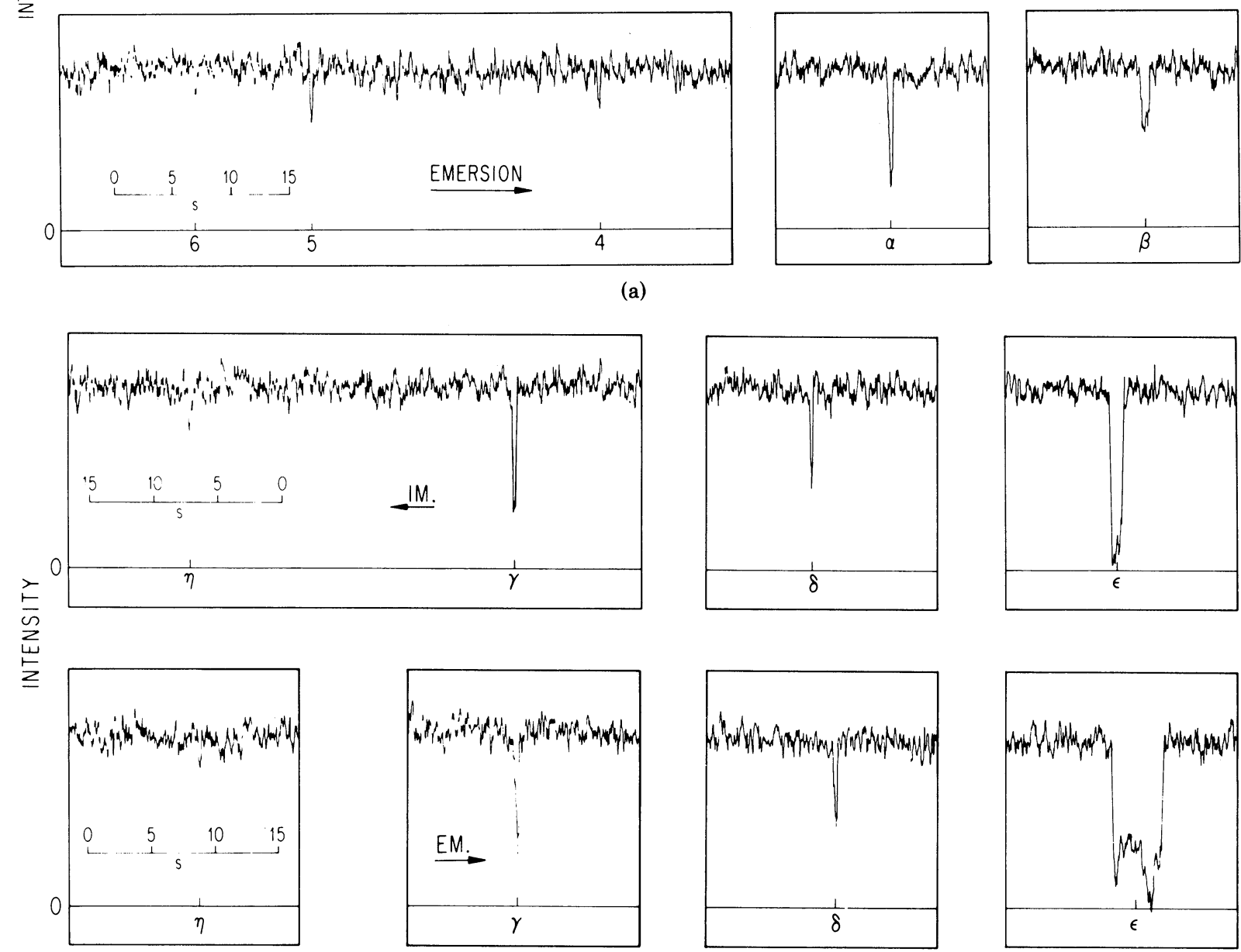

(b)

FIG. 2. Excerpts from the strip-chart recording of the 10 April 1978 occultation, showing all identified ring occultations. The time axis of the emersion records has been reversed, so that they may be more readily compared with the immersion records above them. Occultation times, as indicated by the tick marks, are given in Table II. Note that the $\epsilon$ ring occultations were, in reality, not symmetrically placed in time with respect to the other occultations.

etary occultation profiles, except perhaps to determine average atmospheric scale heights.

\section{ANALYSIS}

\section{a) Overall Ring Geometry}

Because only a single set of observations of the 10 April 1978 occultation is available, it is not possible to solve internally for corrections to the star's position or to Uranus' ephemeris, and hence perform an independent calculation of ring radii, etc. However, if the $\gamma$ and $\delta$ rings are, in fact, circular, as suggested by the March 1977 occultation (Elliot et al. 1978), then their known radii can be used to solve for the position of the star relative to the path of Uranus across the sky.

As a check on the assumption of circularity, the midtime for each pair of ring occultations is given in Table III: A set of concentric circles (or similar, aligned 
TABLE II. Ring occultation observations.

\begin{tabular}{|c|c|c|c|c|c|c|}
\hline \multirow[b]{2}{*}{$\begin{array}{c}\text { Ring } \\
\text { identification }\end{array}$} & \multicolumn{3}{|c|}{ Immersion } & \multicolumn{3}{|c|}{ Emersion } \\
\hline & $\mathrm{h} \quad \begin{array}{l}\mathrm{UT} \\
\mathrm{m}\end{array}$ & $\begin{array}{c}\text { Duration } \\
\text { (s) }\end{array}$ & $\begin{array}{l}\text { Fractional } \\
\text { depth of } \\
\text { occultation }\end{array}$ & $\begin{array}{c}\text { UT } \\
\mathrm{h} \quad \mathrm{m}\end{array}$ & $\begin{array}{c}\text { Duration } \\
\text { (s) }\end{array}$ & $\begin{array}{c}\text { Fractional } \\
\text { depth of } \\
\text { occultation }\end{array}$ \\
\hline 6 & $5: 32: 49.6^{a}$ & $0.3^{\mathrm{a}}$ & 0.20 & $6: 12: 28.5$ & 0.3 & 0.20 \\
\hline 5 & $5: 32: 26.5$ & 0.3 & 0.35 & $6: 12: 38.4$ & 0.3 & 0.35 \\
\hline 4 & $5: 32: 09.8$ & 0.3 & 0.25 & $6: 13: 03.0$ & 0.3 & 0.25 \\
\hline$\alpha$ & $5: 29: 57.5$ & 0.5 & 0.60 & $6: 15: 12.2$ & 0.5 & 0.75 \\
\hline$\beta$ & $5: 29: 01.4$ & 0.9 & 0.40 & $6: 16: 10.0$ & 0.8 & 0.40 \\
\hline$\eta$ & $5: 27: 35.3$ & 0.3 & 0.20 & $6: 17: 35.2$ & 0.3 & 0.20 \\
\hline$\gamma$ & $5: 27: 10.3$ & 0.4 & 0.70 & $6: 18: 00.3$ & 0.4 & 0.75 \\
\hline$\delta$ & $5: 26: 32.5$ & 0.3 & 0.50 & $6: 18: 38.0$ & 0.3 & 0.55 \\
\hline$\epsilon$ & $5: 24: 19.0$ & 1.2 & 0.95 & $6: 21: 21.3$ & 4.0 & $0.6-1.0$ \\
\hline
\end{tabular}

a Relative uncertainty in times and durations is $0.1 \mathrm{~s}$.

b Total duration, not FWHM.

central ellipses) with a common inclination should all exhibit the same midtime. Evidently, the $\gamma$ and $\delta$ rings satisfy this requirement to the accuracy of the timing measurements; consequently they define the adopted midtime of $5^{\mathrm{h}} 52^{\mathrm{m}} 35.25^{\mathrm{s}}$ UT. We make the plausible assumption that these two rings are both coplanar and circular. The midtimes of the $\alpha$ and $\beta$ rings are measurably different from the adopted value, indicating either departures of $\gtrsim 10 \mathrm{~km}$ from circularity, or small inclinations relative to $\gamma$ and $\delta$. Of the remaining five rings, only $\eta$ exhibits a midtime consistent with both circularity and zero relative inclination. For the remainder of the analysis, we shall, however, assume that all of the rings are coplanar (though not necessarily circular), and furthermore, that they lie in the common orbital plane of Uranus' five satellites.

From Earth-Uranus geometry as given in the American Ephemeris and Nautical Almanac, together with mean radii for the $\alpha, \beta, \gamma$, and $\delta$ rings calculated by Elliot et al. (1978) from the 1977 observations, and the timing data in Table II, the projected close-approach distance of the center of each ring to the star is determined. The adopted topocentric velocity of Uranus, projected on the sky, is $20.056 \mathrm{~km} / \mathrm{s}$. The results are given in the fourth column of Table III. The consistency of the results for $\beta, \gamma$, and $\delta$ shows that our observations of these three rings are consistent with their mean radii as determined by Elliot $e t$ al. The discrepant result for the $\alpha$ ring suggests that it departs from circularity by at least $40 \mathrm{~km}$.

Finally, the average close-approach distance of 30985 $\mathrm{km}$ defined by the $\beta, \gamma$, and $\delta$ rings is used with the timing data to calculate the positions in the ring plane of all of the occulting ring segments. The results are given in Table IV. The last two columns of Table IV give, for comparison, the mean radii derived by Elliot et al. (1978). It is emphasized that our absolute radii depend, through the close-approach distance, on the radii of $\beta$, $\gamma$, and $\delta$ adopted by these workers. Any systematic error in these adopted radii will thus also be present in the radii in Table IV. The ring widths are discussed in Sec. IV $b$.

As indicated in Sec. III, a comparison of the radii in Table IV shows that the nine rings observed on 10 April 1978 may indeed be identified with the nine rings reported by Elliot et al. (1978). However, the large variation in radii for rings $6(180 \mathrm{~km})$ and $5(135 \mathrm{~km})$ compared with their mutual separation of $300-400 \mathrm{~km}$ throws some doubt on their existence as two complete rings, rather than as a collection of incomplete arcs. Aside from the well-known $\alpha, \beta, \gamma, \delta$, and $\epsilon$ rings, rings 4 and 5 have also been reported by Millis et al. (1977), and rings $\eta$, 4, and 5 by Bhattacharyya and Bappu (1977) (as their spikes Nos. 4, 5, and 6 respectively).

TABLE III. Midtimes and close-approach distances.

\begin{tabular}{clll}
\hline \hline Ring & $\begin{array}{c}\text { Occultation } \\
\text { midtime (UT) } \\
\mathrm{h} \mathrm{m} \mathrm{s}\end{array}$ & $\begin{array}{c}\text { Assumed } \\
\text { radius }(\mathrm{km}) \\
\text { (Elliot } \text { et } \text { al. } \\
1978)\end{array}$ & $\begin{array}{c}\text { Close approach } \\
\text { distance }(\mathrm{km})\end{array}$ \\
\hline 6 & $5: 52: 39.0 \pm 0.1^{\mathrm{a}}$ & & \\
5 & $5: 52: 32.4$ & & $31018 \pm 3$ \\
4 & $5: 52: 36.4$ & 44844 & $30988)$ \\
$\alpha$ & $5: 52: 34.8$ & 45799 & 30985 \\
$\beta$ & $5: 52: 35.7$ & & 30981 \\
$\gamma$ & $5: 52: 35.2$ & 47746 & average $=30985$ \\
$\delta$ & $5: 52: 35.3^{\mathrm{b}}$ & 48423 & \\
$\epsilon$ & $5: 52: 35.2^{\mathrm{b}}$ & & \\
\hline \hline
\end{tabular}

a Relative uncertainty.

b Adopted midtime $=5: 52: 35.25$. 
TABLE IV. Ring radii, azimuths, and radial widths. Radius calculated in orbital plane of satellites, assuming close-approach distance of $30985 \mathrm{~km}$. Azimuth measured in a prograde direction from ascending node of ring on Earth equator of date. Widths are radial, assuming zero thickness, and are not corrected for diffraction and instrumental response time.

\begin{tabular}{|c|c|c|c|c|c|c|c|c|}
\hline \multirow[b]{2}{*}{ Ring } & \multicolumn{3}{|c|}{ West side } & \multicolumn{3}{|c|}{ East side } & \multicolumn{2}{|c|}{$\begin{array}{c}\text { Mean radius (Elliot } e t \\
a l ., 1978)\end{array}$} \\
\hline & $\begin{array}{l}\text { Radius } \\
(\mathrm{km})\end{array}$ & Azimuth & $\begin{array}{l}\text { Width } \\
(\mathrm{km})\end{array}$ & $\begin{array}{l}\text { Radius } \\
(\mathrm{km})\end{array}$ & Azimuth & $\begin{array}{l}\text { Width } \\
(\mathrm{km})\end{array}$ & $\begin{array}{l}\text { West } \\
\text { side }\end{array}$ & $\begin{array}{l}\text { East } \\
\text { side }\end{array}$ \\
\hline 6 & $42002 \pm 4$ & $53^{\circ} .16 \pm 0.01$ & $\sim 5$ & $42123 \pm 4$ & $137^{\circ} .21 \pm 0^{\circ} .01$ & $\sim 5$ & 42017 & 41943 \\
\hline 5 & 42370 & 52.61 & $\sim 5$ & 42280 & $137^{\circ} .44$ & $\sim 5$ & 42415 & 42304 \\
\hline 4 & 42638 & $52^{\circ} 22$ & $\sim 5$ & 42675 & $138^{\circ} 02$ & $\sim 5$ & 42683 & 42643 \\
\hline$\alpha$ & 44827 & $49^{\circ} .28$ & 9 & 44814 & $140^{\circ} 88$ & 9 & 44834 & 44867 \\
\hline$\beta$ & 45789 & 48.12 & 16 & 45805 & $142^{\circ} .08$ & 14 & 45807 & 45780 \\
\hline$\eta$ & 47299 & $46^{\circ} .44$ & $\sim 5$ & 47299 & $143^{\circ} 74$ & $\sim 5$ & 47323 & 47322 \\
\hline$\gamma$ & 47745 & $45^{\circ} .97$ & 7 & 47747 & $144^{\circ} 22$ & 7 & 47747 & 47745 \\
\hline$\delta$ & 48425 & $45^{\circ} .28$ & $\sim 5$ & 48425 & $144^{\circ} 91$ & $\sim 5$ & 48425 & 48421 \\
\hline$\epsilon$ & 50881 & $42^{\circ} .98$ & 21 & 51439 & $147^{\circ} .69$ & 72 & 51703 & 51056 \\
\hline
\end{tabular}

Radii calculated for rings 4 and $\alpha$ differ by up to 45 and $53 \mathrm{~km}$, respectively, suggesting eccentricities e $\gtrsim$ 0.0005 , or equivalent small relative inclinations. The $\beta$ ring shows a somewhat smaller range in radius of $27 \mathrm{~km}$ (e $\gtrsim 0.0003$ ), while the $\gamma$ and $\delta$ rings are essentially circular. All of these results are quite consistent with the conclusions of Elliot et al. (1978). However, the $\eta$ ring has appeared circular on both 10 March 1977 and 10 April 1978 , but with radii differing by $\sim 23 \mathrm{~km}$. Elliot et al. have also reported a width of $\sim 50 \mathrm{~km}$ for this ring, at variance with our estimate of $\leq 5 \mathrm{~km}$. (See Sec. IV $b$ below). The $\epsilon$ ring is discussed separately in Sec. IV $d$.

The apparent path of the star projected on the ring plane had a minimum radius of $\sim 31000 \mathrm{~km}(\sim 5000 \mathrm{~km}$ above the planet's atmosphere) and maximum radii of $69400 \mathrm{~km}$ on the west side and $107500 \mathrm{~km}$ on the east side. For comparison, the innermost known satellite, Miranda, has a semimajor axis of $130000 \mathrm{~km}$, and Uranus' Roche limit lies at $\sim 70000 \mathrm{~km}$.

The analysis of the single 4 April 1978 ring occultation is deferred to Sec. IV $c$.

\section{b) Ring Widths and Profiles}

The observed radial widths of the ring segments projected onto the satellite orbital plane are given in Table IV. These widths have not been corrected for any of the following important broadening mechanisms:

(1) finite time resolution of the system electronics (equivalent to $\sim 2 \mathrm{~km}$ );

(2) diffraction of starlight around the edges of the rings (Fresnel scale $\sim[\lambda$ (distance from Earth to Uranus) $]^{1 / 2}=2.4 \mathrm{~km}$ );

(3) finite angular diameter of the star [equivalent to $\sim 0.6 \mathrm{~km}$ at Uranus, as estimated from the $B-V$ color and $V$ magnitude given by Liller (1977)].

To examine the effect of (1) and (2), the dominant factors for this occultation, the model profile shown in Fig. 3 was computed. The model consists of an opaque ring $5 \mathrm{~km}$ wide, whose observed profile is modified by diffraction at a wavelength of $2.2 \mu \mathrm{m}$ and smoothed with an exponential time constant of $0.1 \mathrm{~s}$. No correction for the 0.4- $\mu \mathrm{m}$ spectral bandwidth of the observations has been included. This model profile, with a width of $8 \mathrm{~km}$ and a relative depth of 0.85 , is a reasonable representation of the $\alpha, \gamma$, and $\delta$ profiles, if allowance is made for some variation in true widths and opacities. Millis and Wasserman (1978) reported apparent widths of $\sim 8 \mathrm{~km}$ for $\gamma$ and $\delta$ in December 1977, broadened largely by the effects of diffraction at $\lambda=0.86 \mu \mathrm{m}(\sim 1.5 \mathrm{~km})$ and the stellar diameter $(\sim 1 \mathrm{~km}$ at Uranus).

The $\beta$ ring is clearly resolved in the 10 April 1978 data (see Fig. 2), and has an intrinsic width of $\sim 15 \mathrm{~km}$. This is consistent with Millis, Wasserman, and Birch's (1977) measurement of $\sim 1.5 \mathrm{~s}$ for the duration of the $\beta$ occultation on 10 March 1977. Elliot, Dunham, and Mink's (1977) estimates of $\sim 1 \mathrm{~s}$ (implying $\sim 10$-km width) for the $\alpha, \beta, \gamma$, and $\delta$ occultation durations were severely affected by the comparatively large angular diameter ( $\sim 6 \mathrm{~km}$ at Uranus) of SAO 158687. This large size does, however, place a useful lower limit of $\sim 2 \mathrm{~km}$ on the

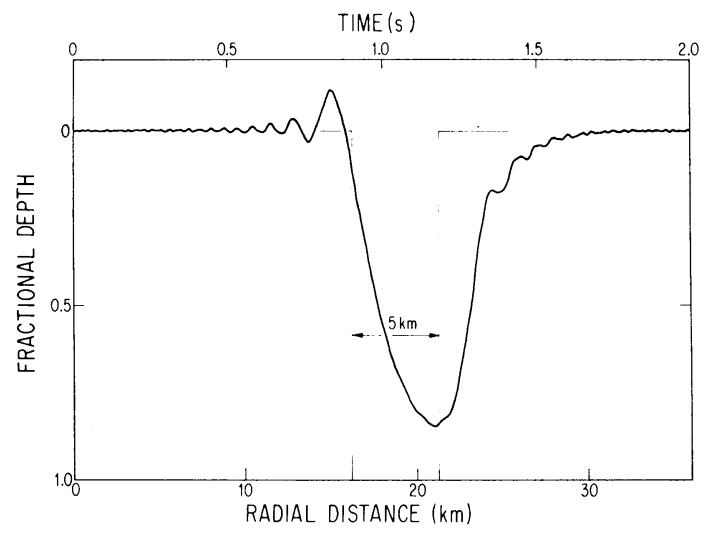

FIG. 3. Theoretical $2.2-\mu \mathrm{m}$ occultation profile of a $5-\mathrm{km}$-wide, opaque ring at the distance of Uranus. The profile was obtained by computing the appropriate Fresnel diffraction pattern, assuming an infinitely distant point source of starlight, and passing this spatial pattern at a velocity of $18 \mathrm{~km} / \mathrm{s}$ through a filter with an exponential time constant of $0.1 \mathrm{~s}$. This filter approximately simulates the response of the system electronics. 
width of any ring which obscured a significant fraction of the starlight, as the $\alpha, \beta, \gamma$, and $\delta$ rings did.

We conclude that rings $\alpha, \gamma$, and $\delta$ are probably quite opaque and have widths of $2-5 \mathrm{~km}$. The optical depth and width of the $\beta$ ring are $\sim 0.5$ and $\sim 15 \mathrm{~km}$, respectively. The widths of rings $\eta, 4,5$, and 6 evidently lie in the range $0.5-5 \mathrm{~km}$, the lower limit being set by the occultation depths and the angular diameter of star No. 5. Their optical depths are, at present, indeterminate.

The only published observation at odds with these conclusions is that of Elliot et al. (1978), who reported a width of $\sim 50 \mathrm{~km}$ for the $\eta$ ring in March 1977, already alluded to in Sec. IV $a$. There is no evidence in Fig. 2 for such a broad feature at the location of the $\eta$ ring.

\section{c) The 4 April 1978 Ring Occultation}

The radius of the single ring identified in the 4 April 1978 occultation data can be estimated from the timing observations and an assumed radius of Uranus. The projected topocentric velocity of Uranus relative to star No. 4 was $18.466 \mathrm{~km} / \mathrm{s}$, which gives a planetary occultation chord of $49640 \pm 190 \mathrm{~km}$. Elliot et al. (1978) determined a radius of $26200 \mathrm{~km}$ for Uranus from the 10 March 1977 occultation. The derived radius of the occulting material is then $50670 \pm 220 \mathrm{~km}$, where the uncertainty represents mainly the 10 -s uncertainty in the midtime of the planetary occultation. The oblateness of Uranus, which is of the order of 0.01 (Danielson, Tomasko, and Savage 1972), has been neglected as it introduces corrections to this radius of only $\sim 20 \mathrm{~km}$. A radial width of the ring segment of $20 \pm 10 \mathrm{~km}$ follows from the $\sim 1$ s duration of the occultation.

Evidently, these observations refer to the $\epsilon$ ring, and the derived radius and width are reasonably consistent with the observations obtained six days later (at the 10 April 1978 occultation immersion) for a nearby part of that ring.

\section{d) The $\epsilon$ Ring}

The most interesting of the rings of Uranus is the outermost $\epsilon$ ring. On 10 March 1977, the two observed segments of this ring exhibited different, well-resolved widths, and, if the ring is coplanar with the others, radii that differed by $\sim 700 \mathrm{~km}$ (Elliot, Dunham, and Mink 1977; Elliot et al. 1978). On 23 December 1977, Millis and Wasserman (1978) observed the $\epsilon$ ring to have intermediate width and radius. In this instance, only preimmersion observations were obtained. The observations on 10 April 1978 now add two more points to this data set, and the noisy 4 April 1978 data provide another, less accurate, point. These six observations of radial width and radius of the $\epsilon$ ring are plotted in Fig. 4, and show a linear relation down to a width of $\sim 20 \mathrm{~km}$. Any inclination of $\lesssim 10^{\circ}$ relative to the other rings would not significantly change the calculated widths; neither are the widths appreciably affected by diffraction or the other broadening effects discussed in Sec. IV $b$.

In light of the relation exhibited in Fig. 4, the concept of the $\epsilon$ ring as a set of fragmentary circular arcs (e.g., Dermott and Gold 1977) is clearly untenable. Furthermore, it now seems highly unlikely that the apparent variation in radius can be explained by an inclined circular ring (e.g., Millis and Wasserman 1978), since this would require a rather fortuitous combination of varying width around the ring and Earth-Uranus observational geometry. The linear relation is, however, consistent with an elliptical ring, coplanar with the other rings, whose inner and outer boundaries are two Keplerian ellipses with slightly different semimajor axes and eccentricities. A model of this type which accurately fits the observations is described in Sec. IVe.

Further information on the nature of the $\epsilon$ ring is provided by a comparison of occultation profiles obtained at different times. Figure 5 shows the 99-km-wide immersion profile obtained by Millis, Wasserman, and Birch (1977) on 10 March 1977, and the 72-km-wide emersion profile of 10 April 1978. The two profiles are very similar, even in the finer details of the structure. Although the optical depths appear to be comparable, no correction has been made to the 10 April 1978 profile to allow for the $\sim 10 \%$ contribution of the planet and rings to the observed flux. Such a correction would increase the optical depth for this occultation by $\sim 25 \%$, and thus support the conclusion of Elliot et al. (1978) that the integrated optical depth of the $\epsilon$ ring is independent of its width. Figure 5 also shows that the edges of the ring remain sharp, even at improved resolution.

The similarity of these two profiles, observed 13

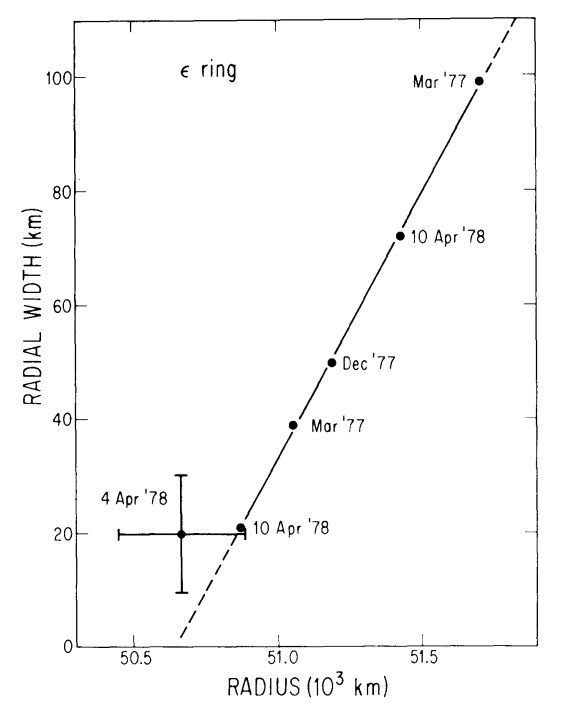

FIG. 4. Radial width plotted against radius for the $\epsilon$ ring for the six occultations observed to date. The multiple observations obtained on 10 March 1977 are represented as two average points. The uncertainty of the 4 April 1978 point is discussed in the text. Error limits on the other points are only slightly larger than the points themselves. The radius scale could contain a systematic error of $\sim 100 \mathrm{~km}$. 

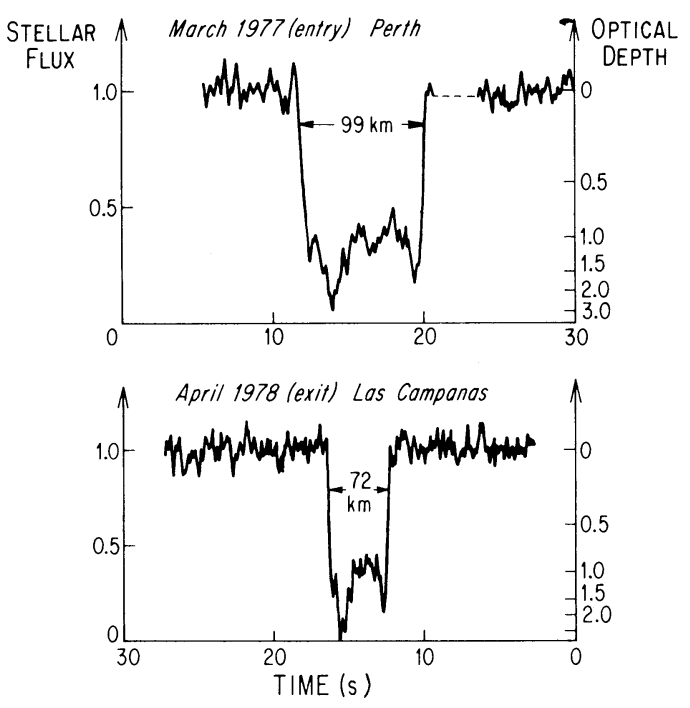

FIG. 5. Comparison of two wide profiles of the $\epsilon$ ring, obtained on 10 March 1977 (Millis, Wasserman, and Birch 1977) and 10 April 1978. Differing width scales are due to different projected velocities of Uranus relative to the two stars. A temporary loss of data on 10 March 1977 is indicated by a dashed line. The $~ 10 \%$ contribution by the planet and rings to the observed $2.2-\mu \mathrm{m}$ flux has not been subtracted from the 10 April 1978 profile.

months apart, is rendered truly remarkable when the orbital and precessional motion of the ring particles is considered. The orbital period of the particles is $8.4 \pm$ $0.1 \mathrm{~h}$, the uncertainty corresponding to the observed range of radii, so that each particle has completed $\sim 1100$ orbits in the intervening time. Differential Keplerian motion between the inner and outer edges of the ring (i.e., $\Delta r / r \sim 50 \mathrm{~km} / 50000 \mathrm{~km}$ ) amounts to at least one complete revolution. Differential precession of the apsidal lines of the elliptical particle orbits (or of the nodal lines of circular orbits) would be expected to further smear and broaden the ring, although it is possible that interparticle collisions act to prevent this and maintain instead a uniform average precession rate. Despite these motions, negligible change has occurred in the shape of the ring profile.

\section{e) A Model of the $\epsilon$ Ring}

An attempt was made to construct an elliptical model of the $\epsilon$ ring which would satisfy the available observations. The simplest physically plausible model consists of a Keplerian ellipse which lies in the satellites' orbital plane, and whose apsidal line precesses due to the oblateness of Uranus. [Dr. Guiseppe Colombo (private communication) first demonstrated that such an ellipse, given the right precession rate, is a reasonable representation of the observations.] As discussed above, the observed profiles and widths of the $\epsilon$ ring suggest that the whole ring precesses as a unit. We therefore chose to fit the model to the centerline of the ring, rather than to the outer and inner edges separately.
Four parameters were adjusted - the semimajor axis, eccentricity, orientation, and apsidal precession rate- - to give the best least-squares fit to the observed radii and azimuths of the $\epsilon$ ring. Figure 6 presents the ten available data points and the best-fitting model. The data are taken from Table 7 of Elliot et al. (1978) (using the average of their radii for the inner and outer edges of the ring), Millis and Wasserman (1978), and Table IV. The 4 April 1978 point is of much lower accuracy than the others and has been omitted from the fit. Also, numerous attempts to match all four 10 March 1977 immersion points failed; only their average location could be fitted. For the final fit, shown in Fig. 6, the Kavalur and Peking points at radii of 51713 and $51740 \mathrm{~km}$ were omitted, and only the Perth and KAO points at $\sim 51678 \mathrm{~km}$ were used. [If the Peking occultation time reported (New Sci. 1977) referred to the beginning of the 9.5-s occultation, rather than to the central time as assumed by Elliot $e t$ al. (1978), then the corresponding radius should be reduced by $\sim 52 \mathrm{~km}$, bringing the point into excellent agreement with the model. Several different times have been reported for the Kavalur $\epsilon$ ring occultation (Bappu 1977; Bhattacharyya and Kuppuswamy 1977a, 1977b; Bhattacharyya and Bappu 1977; Elliot et al. 1978) and conceivably some error remains in the time adopted by Elliot et al. . . This omission makes little difference to the residuals of the other five points, and produces only minor changes in the model parameters.

The elements of the model ellipse for epoch $10 \mathrm{March}$ 1977, $20^{\mathrm{h}}$ UT (JD 2443213.33 ) are

$$
\begin{aligned}
& \bar{a}(\text { semimajor axis })=51284 \pm 6 \mathrm{~km}, \\
& \bar{e}(\text { eccentricity })=(7.80 \pm 0.12) \times 10^{-3}, \\
& \text { Azimuth of periapse }=212^{\circ} \pm 2^{\circ}, \\
& \dot{\tilde{\omega}}(\text { apsidal precession rate })=1^{\circ} .374 \pm 0.006 \\
& \text { day }^{-1} \text {. }
\end{aligned}
$$

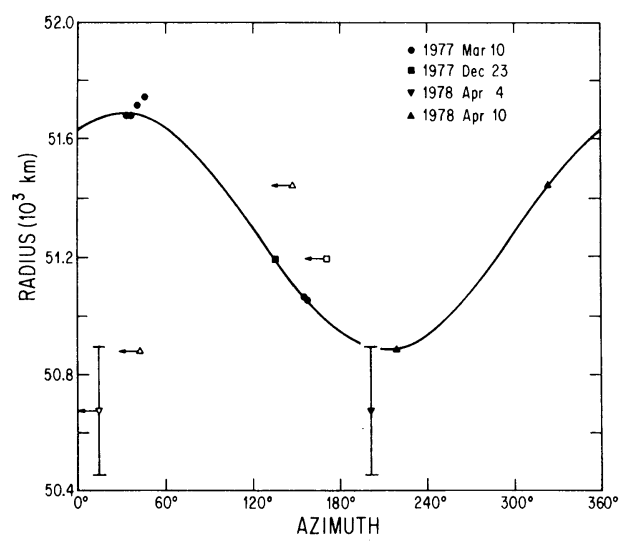

FiG. 6. Observed radius versus azimuth for the $\epsilon$ ring, and the precessing Keplerian ellipse model which best fits these data. Open symbols represent the observed points, while the corresponding filled symbols at the same radii represent these same points corrected to a common epoch (10 March 1977, 20 UT) by removal of precession. The arrows indicate the direction in which the observed points were moved. 
Azimuth is measured in the ring plane, assumed to be the satellite orbit plane, in the prograde direction from the - ascending node of this plane on the earth equator of date. The rms deviation in radius of the seven data points from the model is $7 \mathrm{~km}$, well within the uncertainties. Since this solution might not be unique, initial estimates of $\dot{\tilde{\omega}}$ covering the range $1^{\circ}-10^{\circ} \mathrm{day}^{-1}$ were used in the least-squares analysis. Ten more solutions were discovered in this manner, but all exhibited rms deviations $\geq 26$ $\mathrm{km}$, and typically $\sim 75 \mathrm{~km}$. The validity of this model for the $\epsilon$ ring can be readily tested by future occultations, since the apparent precision of $\dot{\tilde{\omega}}$ should permit extrapolation of the precession for at least ten years.

Combination of the elements $\bar{a}$ and $\bar{e}$ determined above with the width-radius relation of Fig. 4 leads to elements for the ellipses which form the outer and inner edges of the $\epsilon$ ring. If these ellipses have semimajor axes and eccentricities $\bar{a} \pm \Delta a$ and $\bar{e} \pm \Delta e$, the radial width $W$ of the ring, as a function of central radius $\bar{r}$ is given to first order in $\bar{e}$ by

$$
W=2 \Delta a+2(\Delta a / \bar{a}+\Delta e / \bar{e})(\bar{r}-\bar{a}) .
$$

Fitting this linear relation to Fig. 4 yields

$$
\begin{gathered}
\Delta a=30 \mathrm{~km}, \\
\Delta e=0.36 \times 10^{-3} .
\end{gathered}
$$

Finally, Uranus' $J_{2}$ (the dimensionless second gravitational harmonic coefficient), can be derived from the apsidal precession rate, since contributions to $\dot{\tilde{\omega}}$ by satellite perturbations should be negligible. Assuming a planetary equatorial radius of $26200 \mathrm{~km}$ (Elliot et al. 1978), we obtain

$$
J_{2}=(3.43 \pm 0.02) \times 10^{-3} .
$$

The quoted uncertainty is a formal probable error associated with the least-squares fitting procedure; it does not allow for any systematic errors in the occultation analyses. It is assumed that the $\epsilon$ ring is indeed a freely precessing Keplerian ellipse, unaffected by resonant interactions. Subject to this assumption, this value of $J_{2}$ can be used to predict apsidal precession rates for the other supposed elliptical rings:

and

$$
\begin{aligned}
& \dot{\tilde{\omega}}_{4}=2^{\circ} .61 \mathrm{day}^{-1}, \\
& \dot{\tilde{\omega}}_{\alpha}=2^{\circ} .20 \mathrm{day}^{-1},
\end{aligned}
$$

$$
\dot{\tilde{\omega}}_{\beta}=2^{\circ} .04 \mathrm{day}^{-1}
$$

When reduced to a common epoch with these precession rates, the available radius-azimuth data for these three rings are consistent with elliptical models. The $\alpha$ ring data show a variation in radius much larger than the uncertainties and are well distributed in orbital phase, defining an elliptical model with the elements

$$
a=44839 \pm 1 \mathrm{~km}
$$

$$
e=(0.63 \pm 0.03) \times 10^{-3}
$$

azimuth of periapse $=325^{\circ} \pm 2^{\circ}($ epoch 10 March 1977 , $20^{\text {h }}$ UT).

The rms deviation in radius of the eight points from this model is $1.6 \mathrm{~km}$. Models for rings 4 and $\beta$ are less well defined, because of poor orbital phase distribution of the data.

Previous estimates of $J_{2}$ have been based on determinations of the apsidal precession of the satellites Ariel (Dunham 1971) and Miranda (Whitaker and Greenberg 1973), which yielded values of $12 \times 10^{-3}$ and $5 \times 10^{-3}$, respectively. Such determinations of $J_{2}$ are complicated by the effects of the satellites' mutual interactions.

\section{CONCLUSIONS}

(1) The nine rings observed on 10 April 1978 are the same as those described by Elliot et al. (1978) in their analysis of the 10 March 1977 observations. No additional rings were observed, out to a radius limit of 107 $000 \mathrm{~km}$, and no evidence was found for background absorption exceeding $5 \%$.

(2) Observations of the $\eta, \gamma$, and $\delta$ rings are consistent with these three rings being coplanar and circular, although the present $\eta$-ring data are inconsistent in both radius and width with the March 1977 results (Elliot et al. 1978).

(3) Minimum eccentricities for rings $4, \alpha$, and $\beta$, based on the 1977 and 1978 occultations, are $0.0005,0.0005$, and 0.0003 , respectively, under the assumption that all of the rings are coplanar.

(4) Calculated radii for rings 5 and 6 exhibit ranges of $\sim 150 \mathrm{~km}$, comparable to the spacing between these two rings. Further occultation observations are necessary to sort out the structure of this region.

(5) Occultation profiles suggest that rings $\alpha, \gamma$, and $\delta$ are rather opaque and have radial widths in the range $2-5 \mathrm{~km}$. The $\beta$ ring is clearly resolved, and has an average optical depth of $\sim 0.5$ and a width of $\sim 15 \mathrm{~km}$. The widths of rings $\eta, 4,5$, and 6 probably lie between 0.5 and $5 \mathrm{~km}$, but see (2) above.

(6) The width and radius of the $\epsilon$ ring exhibit a linear relation, which strongly suggests that it is a single, continuous, noncircular ring.

(7) The optical depth profile of the $\epsilon$ ring, including the sharp edges, has remained essentially unchanged in 13 months, despite differential orbital motion and precession.

(8) Observations of the $\epsilon$ ring obtained in March 1977, December 1977, and April 1978 all fit a model of a Keplerian ellipse whose apsidal line is advancing at a rate of $1^{\circ} .37 \mathrm{day}^{-1}$. This rate gives a value of Uranus' $J_{2}$ of $3.43 \times 10^{-3}$, if resonant interactions are unimportant.

The authors gratefully acknowledge the assistance of R. Zinn, M. Aaronson, M. Clark, A. Guerra, F. Peralta, 
and L. Papic in making the Las Campanas observations, and of $\mathrm{J}$. Elias in discussions on the desirability of using the $2.2-\mu \mathrm{m}$ band. We also thank R. Lucinio and $\mathrm{G}$. Forrester. PN and PG acknowledge many useful discussions with G. Colombo on the structure of the rings.
We thank J. L. Elliot, R. L. Millis, and L. H. Wasserman for sending preprints of their unpublished results. This research was supported by NASA Grants NGL 05002-140 and NGL 05-002-207 and NSF Grants AST 76-22676, AST 77-20516, and AST 76-24281.

\section{REFERENCES}

Bappu, M. K. V. (1977). Int. Astron. Union Circ. Nos. 3051 and 3068.

Battacharyya, J. C., and Bappu, M. K. V. (1977). Nature 270, 503.

Bhattacharyya, J. C., and Kuppuswamy, K. (1977a). Nature 267, 332.

Bhattacharyya, J. C., and Kuppuswamy, K. (1977b). Int. Astron. Union Circ. No. 3058.

Churms, J. (1977). Int. Astron. Union Circ. No. 3051.

Danielson, R. E., Tomasko, M. G., and Savage, B. D. (1972). Astrophys. J. 178, 887.

Dermott, S. F., and Gold, T. (1977). Nature 267, 590.

Dunham, D. W. (1971). Ph.D. thesis (Yale U.)

Elliot, J. L., Dunham, E., and Mink, D. (1977). Nature 267, 328.

Elliot, J. L., Dunham, E., Wasserman, L. H., Millis, R. L., and Churms, J (1978). Astron. J. 83, 980.
Joyce, R. R., Pilcher, C. B., Cruikshank, D. P., and Morrison, D. (1977). Astrophys. J. 214, 657.

Klemola, A. R., and Marsden, B. G. (1977). Astron. J. 82, 849.

Liller, W. (1977). Astron. J. 82, 929.

Mahra, H. S., and Gupta, S. K. (1977). Int. Astron. Union Circ. No 3061 .

Millis, R. L., and Wasserman, L. H. (1978). Astron. J. 83, 993.

Millis, R. L., Wasserman, L. H., and Birch, P. V. (1977). Nature 267, 330.

Persson, S. E., Nicholson, P. D., Matthews, K., Goldreich, P., and Neugebauer, G. (1978). Int. Astron. Union Circ. No. 3215.

Whitaker, E. A., and Greenberg, R. J. (1973). Mon. Not. R. Astron. Soc. 165, $15 \mathrm{pp}$.

Zellner, B. (1977). Int. Astron. Union Circ. No. 3058

New Sci. 74, 584 (1977). 